\title{
Puente miocárdico como causa de infarto agudo del miocardio. Descripción de un caso y revisión de la literatura
}

\section{Reporte de un caso}

Prieto Macías, Jorge*, Sánchez Arenas, Rodolfo**, Pinzón Obregón, Carlos Ernesto**, Tinajero Vidales, Martha Susana**

\footnotetext{
- Resumen

- Un puente miocárdico (PM) es una banda de músculo cardiaco que - se encuentra por encima de una arteria coronaria, en lugar de estar - por debajo de la misma (arteria tunelizada). Puede causar isquemia - y, eventualmente, infarto del miocardio. Describimos el caso de un - paciente de 33 años de edad sin antecedente de importancia que - acudió al servicio de Urgencias del Hospital General de Zona No.

- I del Instituto Mexicano del Seguro Social (HGZ I, IMSS), por dolor

- precordial de inicio súbito; se diagnosticó infarto agudo del miocar-

- dio de localización anterior y por estudio angiográfico se documentó

- puente muscular en tercio medio de la arteria descendente anterior

- (ADA). En pacientes jóvenes sin factores de riesgo y sin antecedentes

- previos de cuadros sugestivos de isquemia, que inician con angina 0

- infarto agudo del miocardio, se debe hacer el diagnóstico diferencial

- con los puentes musculares miocárdicos. LUX MÉDICA, AÑO 9, NÚMERO

- MAYO-AGOSTO 2014, PP 31-37
}

Palabras clave. Puente miocárdico, dolor precordial, catete-

\section{Abstract}

A myocardial bridge is a band of heart muscle that lies above a coronary artery, rather than below it (artery tunneled). It can cause ischemia and, eventually, myocardial infarction. We describe the case of a 33-year-old pacient without antecedent of importance who came to the emergency room of the General Hospital of the Institute Mexican of the Social Insurance (HGZ I, IMSS), by sudden onset pain chest. He was diagnosed with acute myocardial infarction of anterior location and by study angiographic documented muscular bridge on the middle third of the descending artery. The differential diagnosis of myocardial muscle bridges should be in young patients without risk factors and no previous history of suggestive pictures of ischemia, angina or acute myocardial infarction. LUX MÉDICA, AÑO 9, NÚMERO MAYO-AGOSTO 2014, PP 31-37

Key words. Myocardial bridge, chest pain, catheterization.

Médico Internista-Cardiólogo, Maestro en Ciencias de la Educación, Jefe de la Unidad Médico Didáctica y Profesor Investigador del Centro de Ciencias de la Salud de la Universidad Autónoma de Aguascalientes.

** Estudiantes de Medicina del Centro de Ciencias de la Salud de la Universidad Autónoma de Aguascalientes.

Fecha de recibido: 26 de mayo 2014

Fecha de aceptación: 21 julio 2014

Correspondencia: M en CE Jorge Prieto Macías, Unidad Médico Didáctica, edificio 101, planta alta. Avenida Universidad \# 940. Código Postal 20131. Aguascalientes, Ags., México Teléfono 01(449) 9108441. Correo electrónico dr_prieto@yahoo.com 


\section{Introducción}

El puente miocárdico ( $P M)$ es una anomalía congénita en la que uno o más haces de miocardio cruzan o envuelven un segmento de una arteria coronaria epicárdica, ésta atraviesa la porción intramural del epicardio por debajo del puente muscular y ocasiona una compresión de la luz de la arteria durante la sístole (aunque puede ser normal en la diástole). ${ }^{1}$

Los puentes miocárdicos fueron identificados inicialmente en autopsias realizadas por Reyman en 1737 y posteriormente descritos por Portmann en 1960 en pacientes a los que se realizó cateterismo. ${ }^{2}$

Generalmente se encuentra ubicado a nivel del tercio medio de la arteria descendente anterior (DA $)^{3-4}$ y el principal hallazgo en la angiografía es la compresión de la arteria coronaria durante la sístole. El grado de obstrucción por el puente muscular coronario depende de factores tales como la ubicación, espesor, longitud del puente muscular, y el grado de la contractilidad cardíaca. ${ }^{5}$

Debido a que el PM es un hallazgo frecuente en autopsia de sujetos normales, se había pensado que es una variación anatómica benigna. Sin embargo, aunque esta malformación está presente al nacer, los síntomas generalmente no se desarrollan antes de la tercera década, por lo cual la razón de esto aún no es clara. ${ }^{5}$ Esta patología aqueja principalmente a pacientes con bajo riesgo para enfermedad arterial coronaria. No obstante, cuando es sintomática, se manifiesta en forma de angina, arritmias cardíacas (taquicardia ventricular y taquicardia supraventricular), infarto agudo de miocardio (IAM) y muerte súbita, estos dos últimos de rara aparición. ${ }^{6-7}$

En Brasil se analizaron los resultados de 3,375 cinecoronariografías realizadas en el período de 2003 a 2007, de las cuales 123 $(3,6 \%)$ tuvieron el fenómeno de constricción sistólica de la arteria DA, con diagnóstico de PM. ${ }^{1}$ Otros estudios han publicado una frecuencia del PM entre 1.5 a 16\% mediante coronariografía y en algunas series de autopsias la frecuencia llega a ser del $80 \% .8,9$

\section{Relevancia clínica}

Aunque la mayoría de los pacientes con PM son asintomáticos, se ha asociado con angina de pecho, isquemia miocárdica aguda, aturdimiento miocárdico, infarto del miocardio, arritmia, bloqueo aurículo ventricular completo, muerte súbita e incluso con insuficiencia ventricular izquierda transitoria. ${ }^{10-11}$

El diagnóstico de un puente miocárdico clínicamente significativo debe considerarse en pacientes con angina que no tienen los factores de riesgo tradicionales y que tienen evidencia de isquemia miocárdica. $^{7}$

Para evaluar la importancia clínica de un puente miocárdico aislado, Kremer et al. revisaron 658 angiogramas coronarios normales 
de pacientes con función ventricular izquierda normal y encontraron que $81(12 \%)$ tenían un puente miocárdico de la arteria coronaria descendente anterior. De estos pacientes, 11 tenían una reducción sistólica del diámetro luminal $>50 \%$ y sólo 15 tuvieron angina típica. ${ }^{12}$

\section{Presentación del caso clínico}

Se trata de un hombre de 33 años de edad, que trabaja como supervisor de maquinaria industrial. Acudió al servicio médico refiriendo dolor en hemitórax izquierdo de ocho horas de evolución, mejorando con la prescripción de analgésicos. Cinco horas más tarde por exacerbación del dolor acude nuevamente al servicio de urgencias, diaforético, con tensión arterial de $110 / 70 \mathrm{mmHg}$, frecuencia respiratoria de 20 por minuto, frecuencia cardiaca 100 por minuto y temperatura $36.5^{\circ} \mathrm{C}$. Un electrocardiograma (ECG) (figura 1 y 2), mostró lesión subepicardica en DI, aVL y de V2 a V6. Por laboratorio se documentó: glicemia de $133 \mathrm{mg} / \mathrm{dL}$, creatinina: $0.9 \mathrm{mg} / \mathrm{dL}, \mathrm{CPK}: 1487, \mathrm{CPK}-\mathrm{MB}$ : 175. Se diagnosticó infarto agudo de miocardio y se indicó trombolisis con Tenekteplase. A las 24 hrs de su ingreso nuevamente presentó dolor precordial y el ECG documentó persistencia de la lesión subepicárdica. Por lo anterior fue trasladado a otro hospital donde se efectuó coronariografía, (figura 3) documentándose puente muscular a nivel del tercio medio de la arteria descendente anterior, el resto del árbol coronario sin lesiones, no hubo ateromatosis obstructiva ni trombos. El ventriculograma mostró hipocinesia anterolateral. A los 30 días de evolución el paciente se mantiene asintomático y se encuentra en programa de rehabilitación.

\section{Discusión}

Los puentes miocárdicos son una banda de tejido muscular que rodea un segmento de una arteria coronaria epicárdica y que afecta casi de manera exclusiva la porción media de la arteria descendente anterior. Su incidencia es variable, con un rango que oscila entre $15 \%$ y $85 \%$ en algunas series. Desde las primeras descripciones de casos, la discusión se ha centrado en si debe ser considerada una condición benigna o si es capaz, por sí misma, de causar isquemia, infarto agudo del miocardio, arritmias o muerte súbita.

De igual forma, se ha llegado a proponer que pudiera tener un rol protector para el desarrollo de aterosclerosis, encontrando placas en los segmentos proximales a los puentes, pero no en la porción intramural. Las causas que avalan esta observación no han sido completamente dilucidadas, pero al parecer guardan relación con las fuerzas de cizallamiento, que son menores en la porción proximal y mayores en el trayecto intramural. ${ }^{13,14}$

La cascada de eventos fisiopatológicos se inicia con la compresión sistólica de la arteria, seguida de una reducción persistente en la recuperación del diámetro de la arteria en diástole, lo que puede estimular la activación plaquetaria y ocasionar vasoespasmo, y originar isquemia.

No todos los puentes miocárdicos producen síntomas, por lo cual se han propuesto algunos factores que podrían relacionarse con la aparición de los mismos, 
como son: la localización, el largo y grosor del trayecto intramural, la dominancia de la circulación coronaria, la frecuencia cardíaca y las condiciones relacionadas con el miocardio, como hipertrofia ventricular $y$ estado de contractilidad ${ }^{5}$. Algunos autores han encontrado que hasta $50 \%$ de los pacientes con puentes miocárdicos sintomáticos tienen enfermedad coronaria. En cuanto a la asociación con infarto agudo del miocardio se ha visto que un factor determinante es la localización proximal de los puentes, por ausencia de circulación colateral, más que el vaso comprometido, que, como ya se mencionó, afecta casi de forma invariable a la descendente anterior, y de manera menos frecuente las ramas diagonales y marginal obtusa, $y$, muy ocasionalmente, la descendente posterior. ${ }^{13,15}$
La forma de presentación va desde pacientes con dolor torácico atípico (hasta $45 \%$, como en el caso de nuestro paciente) o asintomáticos, hasta aquéllos con episodios de angina. Un puente miocárdico se considera significativo desde el punto de vista hemodinámico cuando se alcanza una reducción del diámetro mínimo luminal de $70 \%$ durante sístole y de 35\% durante diástole. ${ }^{13}$

Debe señalarse además, que el puente miocárdico tiene un papel significativo en la muerte súbita cardiaca. En un estudio realizado por Corrado se encontró que de los 16 casos de muerte súbita de origen cardiaco en sujetos menores de 35 años de edad, causadas por enfermedades coronarias no ateroscleróticas, el puente miocárdico se demostró en 6 casos $(37,5 \%)$ en la arteria descendente anterior. ${ }^{16}$

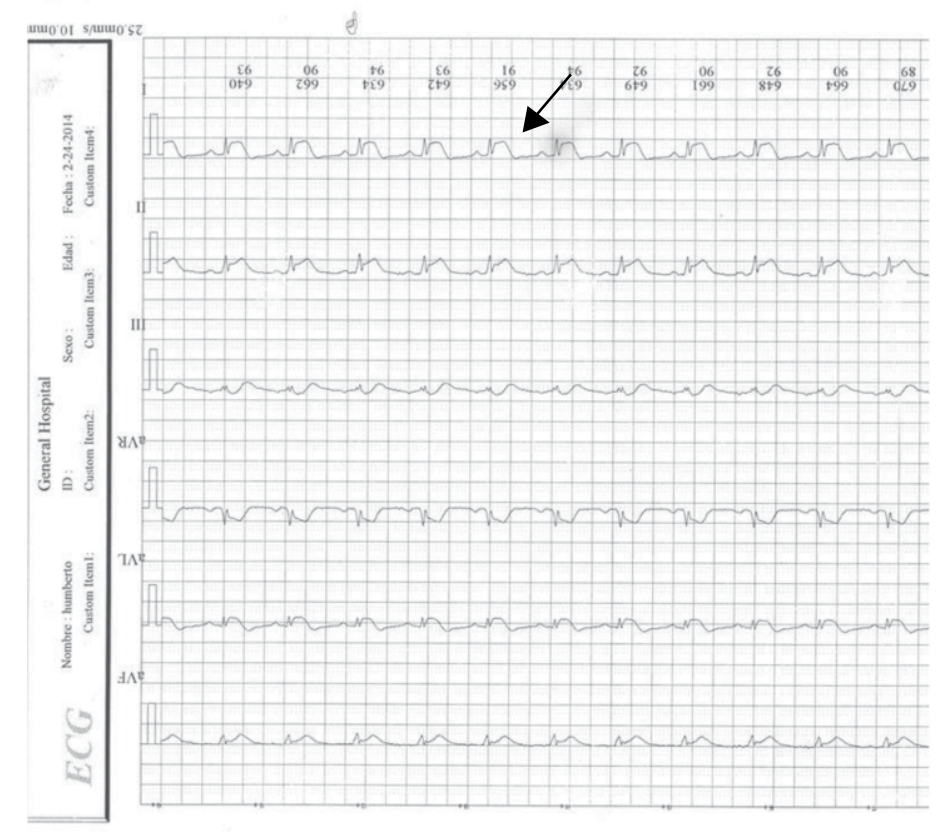

Figura 1. Lesión subepicárdica de DI, aVL 


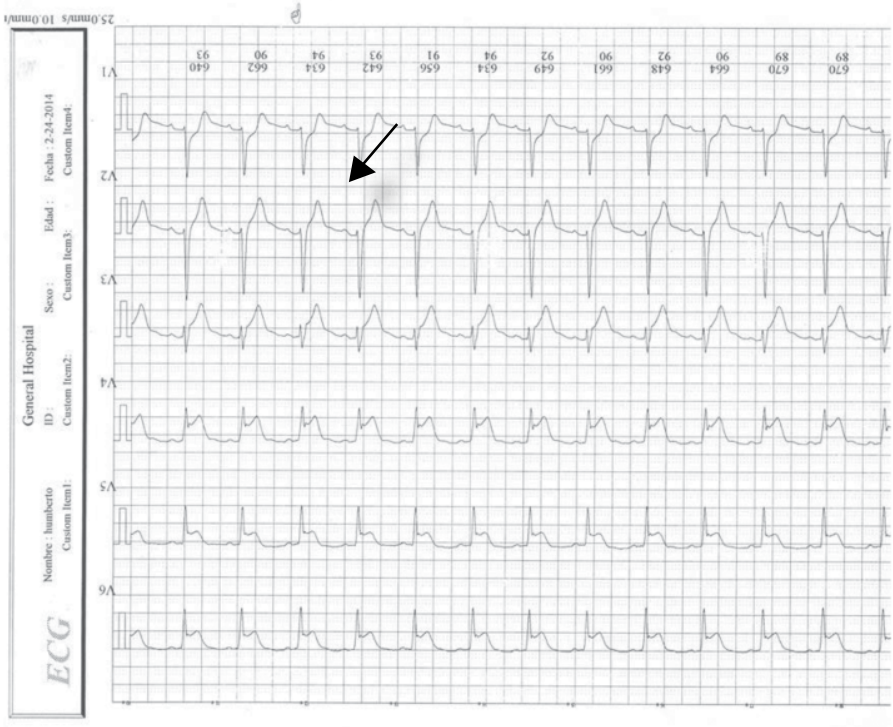

Figura 2. Lesión subepicárdica de V2- V6

\section{Diagnóstico}

Aunque el método diagnóstico de referencia es la coronariografía, existen otras técnicas invasivas que se han utilizado en el diagnóstico, como la ecografía intravascular y el eco-doppler intravascular. ${ }^{2}$ Actualmente, con el advenimiento de la angiografía coronaria por tomografía computarizada multidetector (ACTCM) ha surgido una alternativa no invasiva para la evaluación de las arterias coronarias, que no sólo permite el diagnóstico de la enfermedad coronaria y el grado de estenosis, sino también la detección y la clasificación de anomalías coronarias. ${ }^{17-21}$

En nuestro caso se realizó una angiografía coronaria, en la cual se observa una estrechez en el tercio medio de la arteria descendente anterior, con lo cual se evidencia la presencia del puente miocárdico (figura 3).

Los datos electrocardiográficos de lesión subepicárdica en DI, aVL y de V2 a V6 pueden hacer sospechar en pericarditis; sin embargo, el cuadro clínico, la elevación de enzimas y la imagen de amputación en el primer vector en V1-2 permitió hacer el diagnóstico de infarto agudo del miocardio. 


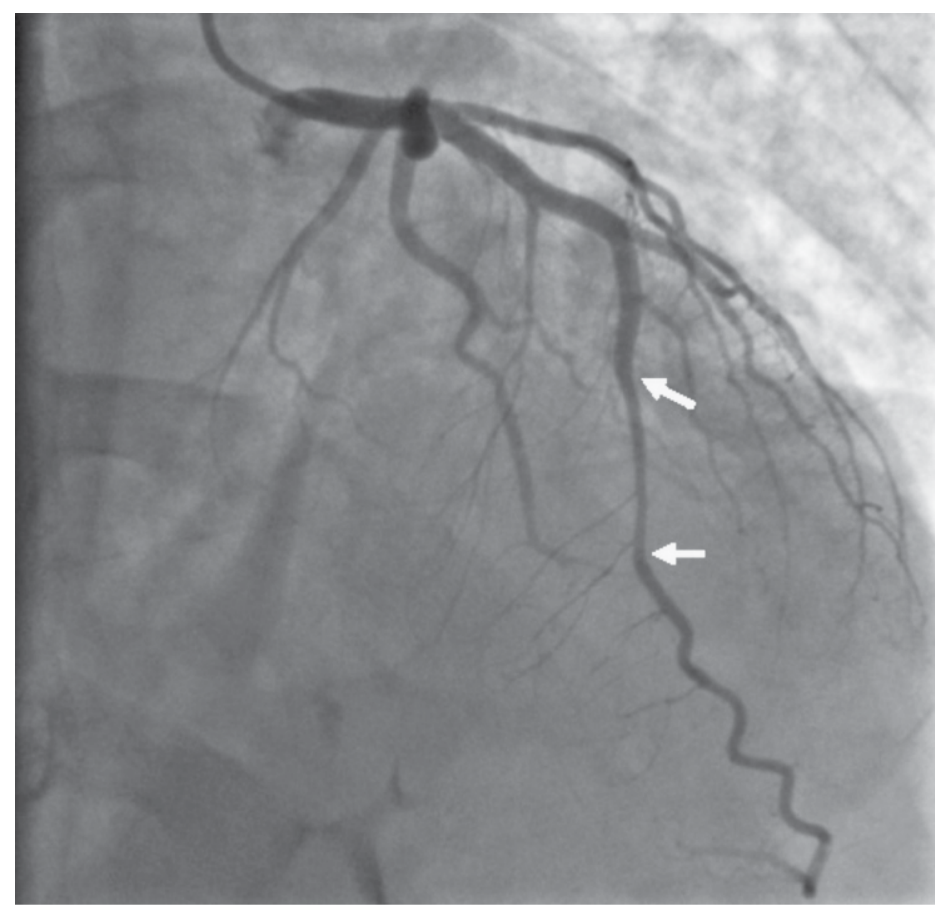

Figura 3. Coronariografía en donde se observa una disminución de la luz de la arteria descendente anterior en su tercio medio debido a la presencia de un puente miocárdico.

Los enfoques terapéuticos que se han intentado para puente miocárdico incluyen bloqueadores beta, bloqueadores de los canales de calcio y puente coronario. ${ }^{22}$
El pronóstico a largo plazo de los puentes musculares aislados o múltiples en la DA es, en la mayoría de las ocasiones, favorable y benigno. ${ }^{23}$

\section{Conclusiones}

Los puentes miocárdicos pueden encontrarse como hallazgo incidental en sujetos asintomáticos u ocasionar angina y estar asociados a alguna enfermedad isquémica coronaria y otras condiciones cardiovasculares. La disponibilidad de nuevas técnicas ha hecho que el diagnóstico sea más oportuno. Las opciones de tratamiento siguen considerando el manejo médico como primera línea, como sucedió con nuestro paciente, y las intervenciones mínimamente invasivas o la cirugía se reservan para algunos casos muy específicos. Finalmente, aún no existen determinantes acerca de la evolución natural de dicha patología, motivo por el cual es de vital importancia el análisis cuidadoso de la situación clínica y funcional de los pacientes de forma individualizada para que, de esta forma, las decisiones tomadas con respecto al diagnóstico y tratamiento puedan llevar al paciente a una mejoría importante con repercusiones mínimas para la vida y/o la función. 


\section{Bibliografía}

1. Pereira B, Peressoni $S$, Menegotto $T$, Do Amaral $M$, Castro P. Puente miocárdico: Evolución clínica y terapéutica. Arq Bras Cardiol, 2010; 94(2):178-184.

2. Carrascosa $P$, López $M$, Capuñay $C$, Deviaggiano $A$, Vallejos J, Carrascosa J. Prevalencia y características de los puentes miocárdicos en estudios de angiografía coronaria por tomografía computarizada multidetector. 2009; Revista Argentina de Cardiología: 77(4).

Disponible en http://www.scielo.org.

ar/scielo.php?script $=$ sci arttext\&pid $=\mathrm{S} 1850-37482009000400006$.

3. Faruqui A, Maloy W, Felner J, Schlant R, Logan W, Symbas P. Symptomatic myocardial bridging of coronary artery. Am J Cardiol 1978;41:1305-1310.

4. Irvin $G$. The angiographic prevalence of myocardial bridging in man. Chest, 1982;81:198-202.

5. Alegría R, Herrmann J., R. Holmes D, Holmes Jr. A, S. Charanjit R. Myocardial bridging. European Heart Journal, 2005;26:1159-1168 doi:10.1093/eurheartj/ehi203.

6. Sociedade Brasileira de Cardiologia. Diretries de doença coronariana crônica e angina estável. Arq Bras Cardiol. 2004; 83 (2): 2-43.

7. Marin M. Tortoledo F, Aguilar P. Puente muscular intramiocárdico como causa de angina intratable. Papel del "IVUS" y del tratamiento endovascular. Sociedad Venezolana de cardiología. Sociedad Venezolana de Available from: http://es.slideshare. net/soveci/puente-muscular-intramiocrdico-comocausa-de-angina-intratable-papel-del-ivus-y-deltratamiento-endovascular.

8. Rossi L, Dander B, Nidasio GP, Arbustini E, Paris B, Vassanelli $C$ et al.Myocardial bridges and ischemic heart disease. Eur Heart J 1980;1:239-245.

9. Geirenger E. The mural coronary. Am Heart J 1951;41:359-368.

10. Tortoledo F, Izaguirre L, Tortoledo A. Puentes miocárdicos de las arterias coronarias. Gac Méd Caracas. 2002;110(4): 474-477.

11. Diefenbach $C$, Erbel R, Treese N, Bollenbach E, Meyer J. Incidence of myocardial bridges after adrenergic stimulation and decreasing afterload in patients with angina pectoris, but normal coronary arteries [German]. Z Kardiol 1994;83:809-815.

12. Kramer JR, Kitazume $H$, Proudfit WL, Sones FM Jr. Clinical significance of isolated coronary bridges: benign and frequent condition involving the left anterior descending artery. Am Heart J 1982;103:283288.
13. Echeverry Eduardo J, Murillo Nelson E, Villadiego Juan S, Ochoa Julián A, Velásquez Jorge G. Puentes miocárdicos como causa de infarto agudo del miocardio Reporte de una serie de once casos y revisión de la literatura. Rev. Colomb. Cardiol. [serial on the Internet]. 2013 Aug [cited 2014 July 22] ; 20( 4 ): 240-245. Available from: http://www.scielo. org.co/scielo.php?script =sci_arttext\&pid=S0120$56332013000400012 \& \operatorname{lng}=e^{-}$.

14. Lozano I, Baz J, López R, Pinar E, Picó F, Valdés $M$, Larman $M$, Martínez J. Pronóstico a largo plazo de los pacientes con trayecto intramiocárdico de la arteria descendente anterior con compresión sistólica. Rev Esp Cardiol. 2002;55(4):359-64.

15. Sociedad Española de Radiología Médica. SERAM 2010.com/modules.php?name = posters \&d op...3. Trayectos coronarios intramiocárdicos: detección 2.

16. Corrado D, Thiene G, Cocco P, Frescura C. Nonatherosclerotic coronary artery disease and sudden death in the young. Br Heart J. 1992; 68: 601-7.

17. Masciarelli I. Evaluación del Puente Miocárdico por la Angiotomografía de las Coronarias. Rev bras ecocardiogr imagem cardiovasc.2013;26(1):2-3. Comentario Editorial.

18. Kantarci M, Duran C, Durur I, Alper F, Onbas O, Gulbaran $M$, et al. Detection of myocardial bridging with ECG-Gated MDCT and multiplanar reconstruction. Am J Roentgenol 2006;186:S391-S394.

19. Ghersin E, Litmanovich D, Dragu R, Rispler S, Lessick J, Ofer A, et al. 16-MDCT coronary angiography versus invasive coronary angiography in acute chest pain syndrome: a blinded prospective study. Am J Roentgenol 2006;186:177-84

20. Ropers D, Rixe J, Anders K, Kuttner A, Baum U, Bautz $W$, et al. Usefulness of multidetector row spiral computed tomography with 64- ? 0.6-mm collimation and $330-\mathrm{ms}$ rotation for the noninvasive detection of significant coronary artery stenoses. Am J Cardiol 2006; 97:343-348.

21. Gómez J, Rodríguez F. Limitaciones Orgánicas y funcionales por el fenómeno de milking. Revista Médica. Disponible en: http://www.medigraphic.com/pdfs/ imss/im-2014/im143t.pdf.

22. Barriales V, Carro A, Fernández M, Morales C. Tratamiento quirúrgico de un puente intramiocárdico de la arteria descendente anterior sintomático. Cardiocore, 2012;47(4):169. doi: 10.1016/j.carcor.2011.12.001.

23. Arnau $M$, y cols. Puente miocárdico coma causa de isquemia aguda. Descripción del caso y revisión de la bibliografía. Rev Esp Cardiol. 1999;52(6):375-380. 